\title{
A Perspective on Chemical Engineering Education
}

\author{
Kamal I Al Malah* \\ Engineering Division Chair, Higher Colleges of Technology,UAE, almalak61@hotmail.com
}

*Corresponding author: Kamal I Al Malah, Engineering Division Chair, Higher Colleges of Technology, Ruwais, Abu-Dhabi, UAE, Email: kalmalah@hct.ac.ae

\author{
Received Date: March 12, 2019
}

Published Date: March 21, 2019

\begin{abstract}
Chemical Engineering education faces new challenges that need to be addressed by chemical engineering educators. The dynamically changing learning environment and that of teaching require the teacher to relax and contemplate. Among all community sectors, educators must be number one in sniffing the smell of change, be willing to adapt, and later be capable to adopt new pedagogical findings, methods and techniques, and means. In brief, the notion of revolutionizing chemical engineering education does not necessarily mean that we should ignore the pillars of it. On the contrary, we should expand in a way to also tackle the latest critical socio-technical issues both on the stem (roots) and branches level. The tree of chemical engineering education has to grow in height (chance to explore new areas and also see the sun) above the ground and have more branches (industrial applications to be tailored to chemical engineering), as well. A strong tree with green leaves and fruits emanates from well-irrigated roots. On the one hand, we need to contemplate about our ancestors in terms of re-visiting fundamental science topics. On the other hand, we need to stay updated amid "continuously changing" learning and teaching environment as far as the latest pedagogical findings, domain knowledge, technology for teaching (i.e., means of dissemination and communication), web-based online resources, lab equipment and tools, relevant software, computational techniques, and programing languages are concerned. Topics like public awareness, soft skill, and innovation and entrepreneurship were also highlighted.
\end{abstract}

Keywords: Chemical engineering; Education; Trends; Soft skills; Environment; Society; Challenges; Awareness; Innovation; Entrepreneurship

\section{Introduction}

Engineering education, in general, and chemical engineering education, in particular, are both dynamic in nature and should always reflect the latest changes taking place on the side of chemical industries in terms of critical problems and challenges being faced or addressed by the people of industry and also in terms of development and aspirations they are looking forward to realizing.

Sternberg [1-3] proposed that there are three key and keen skills that are necessary to create a successful graduate. These are: analytical (or critical), creative and practical skills. Progress and success in life and in professional careers can be achieved through having a balance of analytical (or critical), creative, and practical skills. Such three intellectual activities are regarded as the recipe for success in career choice and development. Developing a multitude of balance of such three skills while at the same time knowing how and when to use each will make students act smarter rather than work harder.
In his report about the global state of the art in engineering education, Graham [4] pointed out that engineering educational features characterizing the 'current leaders' (namely, MIT, Stanford University, Aalborg University and TU Delft) included user-centered design, technology-driven entrepreneurship, active project-based learning and a focus on rigor in the engineering fundamentals; on the other hand, the characterizing features in 'emerging leaders' (namely, Singapore University of Technology and Design, Olin College of Engineering, University College London, the Pontifical Catholic University of Chile and Iron Range Engineering) include work-based learning, multidisciplinary programs and a dual emphasis on engineering design and student self-reflection. Case studies also showed that the 'emerging leader' programs have benefitted from strong and visionary academic leadership, augmented by new tools that support educational exploration and student assessment. 
In terms of challenges facing upgrade of engineering education, Graham [4] gave examples, like reconciliation of government and higher education goals, the challenge of delivering studentcentered active learning to large student cohorts, the monolithic mono- disciplinary structure of many engineering schools, and faculty appointment and promotion systems that are not perceived as rewarding teaching achievement.

In terms of engineering education trends, Graham [4] concluded the following three:

The first is a tilting of the global axis of engineering education leadership from north to south and from high-income countries to the emerging economic 'powerhouses' in Asia and South America. Many among this new generation of world leaders will be propelled by strategic government investment in engineering education as an incubator for the technology-based entrepreneurial talent that will drive national economic growth.

The second is a move towards socially-relevant and outwardfacing engineering curricula. Such curricula emphasize student choice, multidisciplinary learning and societal impact, coupled with a breadth of student experience outside the classroom, outside traditional engineering disciplines and across the world.

The third is the emergence of a new generation of leaders in engineering education that delivers integrated student-centered curricula at scale. The case studies indicate a number of institutions that have developed such a model, where this curricular coherence and integration is delivered through a connective spine of design projects.

Jain [5] outlined the major features (or factors) which will shape out the future of engineering education, namely:

- Hands-on Learning: A blend of both hands-on learning and traditional lecture learning. The hands-on learning programs allow students to use classroom materials in realworld applications such as the 3D printing labs and state-ofthe-art research labs.

- Industry-specific Programs: Industry professionals and professors come together in such programs to discuss innovation, cutting-edge technologies and discoveries.

- Open-door Concept: Open-concept labs to encourage students to take part in brainstorming sessions, group presentations and instructor demonstrations. The desk and chairs are being replaced with tables, laptops, technologyequipped workstations and whiteboards to visualize, design, and map their thought processes.

- $\quad$ E-learning: The textbooks are gradually replaced by adaptive learning software. The course materials can be easily updated with such software to ensure that students learn the most recent course material.

- $\quad$ Enrollment in non-Technical Skills: Engineering students also need to develop non-technical skills, in addition to the main curriculum. Universities and colleges encourage students to enroll in soft-skill courses, like public speaking, professional writing, team player, leadership, and career preparation.

- Innovation and Entrepreneurship: Incubation-centers, warehouses, and fabrication centers are being set up with a help from industry.

The National Academy of Engineering declared, in February 2008, the engineering grand challenges as reported by El Maraghy [6]: "These are:

1) Make Solar Energy Economical; 2) Provide Energy from Fusion; 3) Develop Carbon Sequestration Methods; 4) Manage the Nitrogen Cycle; 5) Provide Access to Clean Water; 6) Engineer Better Medicines; 7) Advance Health Informatics; 8) Secure Cyberspace; 9) Prevent Nuclear Terror; 10) Restore and Improve Urban Infrastructure; 11) Reverse Engineer the Brain; Enhance Virtual Reality; 12) Advance Personalized Learning; 13) Engineer the Tools of Scientific Discovery. Obviously, some of these challenges are imperative for human survival, some will make us more secure against natural and human threats, and all are intended to improve quality of life. They are of course all complex problems and mostly of a global scale. The other thing that they have in common is they are socio-technical complex systems".

Marsithi \& Alias [7] emphasized that engineering graduates having a high employability is a big concern among stakeholders. They correlated successful intelligence, composed of analytical, creative and practical thinking skills, with greater employability potential and also with greater career success. Developing successful intelligence among engineering students through appropriate means is highly appreciated. In their opinion, Problem-Based Learning (PBL) method seems to have the necessary elements that can promote successful intelligence. They subdivided the analytical thinking into gathering information, analyzing, evaluating, problem solving, and decision making step. The creative thinking is mainly made of formulation of a good problem and finding its solution(s). Finally, the practical thinking is dedicated to the use of ideas and analysis in an effective way.

\section{Pedagogical Approach}

In light of the afore-mentioned cited literature and also based on my versatile and multi-dimensional experience in chemical engineering domain both as a student and later as a faculty member, here is my humble input to the process of updating or more dramatically upgrading the engineering education, in general, and chemical engineering education, in particular:

\section{Universal engineer}

Chemical engineering education by itself is more of an applied science than just simply a recipe or know-how technology. This definitely means we cannot compromise the basics or fundamentals that underlie any process/system part of chemical engineering applications. There is always a necessity that a graduate chemical engineer be strong in mastering the conservation laws and basic 
principles that govern, for instance but not limited to, any chemical, biochemical, food, polymer and biopolymer, pharmaceutical, paper, cosmetics, paints, detergents, oil and gas, cement and ceramics, and finally minerals process/system. In this context, chemical engineer is denoted as "universal" engineer in terms of post applications being handled by a chemical engineer in real life.

\section{Back to the roots}

In my opinion, I prefer that a chemical engineer revisits and knows more about his/her ancestors; i.e., chemistry, applied chemistry, and even biochemistry topics. Chemical engineering educators can realize that some hot topics or grand challenges, declared by The National Academy of Engineering [6], require grabbing further fundamental knowledge in materials properties, energy transfer and conversion, reaction chemistry of species and their life cycle and fate in environment, food chemistry, biochemistry, and biology. Again, this brings us back to square number one or perhaps number zero in terms of mastering prerequisite skeletal knowledge.

\section{Chemistry of things}

In light of the above two points, I would rather go with the option of restructuring background chemistry courses, needed prior to entering the world of chemical engineering. Specifically, it is preferred that we start by general chemistry I course, pass by general chemistry II course, and then chemistry courses can be re-designed to handle and accommodate the versatile topics to be later tackled by a chemical engineer. For example, we may suggest the following handy ready-mix background chemistry courses as they pertain to the nature of material (or solution) we deal with. This includes; for example, real solutions and gas mixtures, macroemulsions and micro-emulsions (or, dispersions, colloids, and hydro-colloids), electrolytes, biochemical/biological solutions, solids, semi-solids, viscous fluids, and polymers and biopolymers. Such topics can be combined into two to three courses right after passing the general chemistry II course. To be more specific, we talk about characterization of the afore-mentioned systems and their applications as encountered in chemical industries, in the broad sense.

\section{Innovation and entrepreneurship}

The call for innovation and entrepreneurship skill is worth contemplating. In my opinion, this requires that the student masters (or even PhDs as opposed to masters) well her/his area of specialty before she/he arrives at this advanced intellectual step. Aside from having a genius kid who can attempt the self-learning process and dedicate herself/himself to a topic of choice, which represents the master passion above all others, we talk about a regular average student who needs to be driven by an instructor where the latter will pave the road to the former. As you all know, the main duty of the teacher is to make the learning curve for the student steeper (i.e., faster in learning) and smoother (continuous and sequential) on the student's side. Consequently, I can say that the call for building a student's innovative and entrepreneurial skill at an early stage will be pre-mature for an average regular student.
This can be dealt with at a later stage; i.e., after graduation during a summer internship. To be an innovative and entrepreneurial person, one has to be exposed to so many lab and life experiments, visual demonstrations, and patterns/prototypes so that she/he has a clear vision about the latest trend/approach pursued in a given direction or dimension of development, improvement, re-novation, and finally innovation.

\section{Soft skills}

On the contrary, the call for acquiring non-technical "soft" skills can be in fact initiated at an earlier stage; say at the high school level. There will be a plenty of time when students can learn and exercise such delicate and diligent skills. This will make them readier than they stand now. This will also help them capitalize on the new environment (life at a college or university) in terms of exploiting blended learning methods and expanded/extended relationships with their colleagues and with their instructors, as well.

\section{Public awareness}

The call for public awareness of contemporary societal complex issues or of any other issues related to her/his field of study, I guess we should rely in part on the fact that a chemical engineering student is a member of her/his society and does not live in a jungle (i.e., Tarzan). This means that she/he will be more or less aware of such critical nagging issues via means of social media, Internet, and personal communications. At this point, it is worth saying that that the cyberspace is full of data, information, and knowledge on any topic you may think of. This in fact will make it even a more difficult task for a beginner to dig, search, swallow, and digest the information. Aside from a wrong perception that can be made by the beginner her/himself, the available sources of cyberspace information may contain undocumented or unauthenticated pieces of information, which can be inaccurate, misleading, incomplete, or even false. Again, here comes the role of the teacher to help a student formulate the scientific/mental way of thinking and reasoning toward what she/he sees and hears and also how to mentally filter out such immense and overwhelming data/information.

\section{Super teachers wanted}

By the start of this century, the humanity reached the so-called "mushroom cloud" of information explosion, both in depth and breadth (i.e., in terms of volume and content, respectively) of the massive information being generated by and shared amongst all human kind. This being the case, the teacher cannot stay inanimate but has to be dynamic by instinct. The professor must stay updated in terms of the latest pedagogical findings, adopted technology, domain knowledge, technology for teaching (i.e., means of dissemination and communication), web-based online resources, lab equipment and tools, relevant software, computational techniques, and programing languages.

\section{Conclusion}

In brief, the notion of revolutionizing chemical engineering education does not necessarily mean that we should ignore the pillars of it. On the contrary we should expand in a way to also 
tackle the latest critical socio-technical issues both on the stem (roots) and branches level. The tree of chemical engineering education has to grow in height (chance to explore new areas and also see the sun) above the ground and have more branches (industrial applications to be tailored to chemical engineering), as well. A strong tree with green leaves and fruits emanates from wellirrigated roots. On the one hand, we need to contemplate about our ancestors in terms of re-visiting fundamental science topics. On the other hand, we need to stay updated amid "continuously changing" learning and teaching environment as far as the latest pedagogical findings, domain knowledge, technology for teaching (i.e., means of dissemination and communication), web-based online resources, lab equipment and tools, relevant software, computational techniques, and programing languages are concerned.

\section{Acknowledgement}

None.

\section{Conflict of Interest}

No conflict of interest.

\section{References}

1. Sternberg, RJ (1997) Successful Intelligence: How Practical and Creative Intelligence Determine Success in Life. New York, p. 12.

2. Sternberg RJ (2003) Implications of the Theory of Successful Intelligence for Career Choice and Development. Journal of Career Assessment 11(2): 136-152.

3. Sternberg RJ (2005) The Theory of Successful Intelligence. Interamerican Journal of Psychology 39(2): 189-202.

4. Graham R (2018) The global state of the art in engineering education. MIT, Cambridge, MA 02139, USA.

5. Jain S (2018) \#5 Emerging Trends in Engineering Education in India. Entrepreneur India, an international franchise of Entrepreneur Media. Tula's Institute, Dehradun.

6. El Maraghy WH (2011) Future Trends in Engineering Education and Research. in: G Seliger, et al. (eds.), Advances in Sustainable Manufacturing: Proceedings of the $8^{\text {th }}$ Global Conference 11 on Sustainable Manufacturing.

7. Marsithi A, Alias M (2013) Successful intelligence via Problem-Based Learning: Promoting employability skills of engineering graduates. Proceedings of the Research in Engineering Education Symposium, Kuala Lumpur, Malaysia. 